\title{
Complex Dynamical Behaviors of a Fractional-Order System Based on a Locally Active Memristor
}

\author{
Yajuan Yu, ${ }^{1}$ Han Bao $\left(\mathbb{D},{ }^{2}\right.$ Min Shi $\left(\mathbb{D},{ }^{3}\right.$ Bocheng Bao $\mathbb{D}^{1},{ }^{4}$ Yangquan Chen $\mathbb{D},{ }^{5}$ and Mo Chen $\left(\mathbb{D}{ }^{4}\right.$ \\ ${ }^{1}$ Aliyun School of Big Data, Changzhou University, Changzhou 213164, China \\ ${ }^{2}$ College of Automation Engineering, Nanjing University of Aeronautics and Astronautics, Nanjing 210016, China \\ ${ }^{3}$ Institute of Advanced Technology, Nanjing University of Posts and Telecommunications, Nanjing 210042, China \\ ${ }^{4}$ School of Information Science and Engineering, Changzhou University, Changzhou 213164, China \\ ${ }^{5}$ Mechatronics, Embedded Systems and Automation Lab, School of Engineering, University of California, Merced, Merced, \\ CA 95343, USA
}

Correspondence should be addressed to Bocheng Bao; baobc@cczu.edu.cn

Received 10 May 2019; Revised 30 August 2019; Accepted 8 October 2019; Published 20 November 2019

Guest Editor: Lazaros Moysis

Copyright (c) 2019 Yajuan Yu et al. This is an open access article distributed under the Creative Commons Attribution License, which permits unrestricted use, distribution, and reproduction in any medium, provided the original work is properly cited.

A fractional-order locally active memristor is proposed in this paper. When driven by a bipolar periodic signal, the generated hysteresis loop with two intersections is pinched at the origin. The area of the hysteresis loop changes with the fractional order. Based on the fractional-order locally active memristor, a fractional-order memristive system is constructed. The stability analysis is carried out and the stability conditions for three equilibria are listed. The expression of the fractional order related to Hopf bifurcation is given. The complex dynamical behaviors of Hopf bifurcation, period-doubling bifurcation, bistability and chaos are shown numerically. Furthermore, the bistability behaviors of the different fractional order are validated by the attraction basins in the initial value plane. As an alternative to validating our results, the fractional-order memristive system is implemented by utilizing Simulink of MATLAB. The research results clarify that the complex dynamical behaviors are attributed to two facts: one is the fractional order that affects the stability of the equilibria, and the other is the local activeness of the fractional-order memristor.

\section{Introduction}

Nonlinear electronic circuits provide powerful and analytical platforms for people to realize and understand the complex dynamical behaviors in physics [1]. Chaotic circuits especially have become effective tools for studying chaos theory. The memristor, originally defined as the forth element of the circuit by Chua in 1971 [2], is a nonlinear circuit device besides the nonlinear resistor, capacitor, and inductor. As a result, many novel memristive circuits have been constructed by integrating the memristors with versatile nonlinearities into some existing linear or nonlinear circuits [3-11]. In these memristive circuits, rich dynamical behaviors have been reported and tested by numerical simulations and hardware experiments, such as chaos and hyperchaos [12, 13], hyperchaotic multiwing attractors $[14,15]$, coexisting multiple attractors $[16,17]$, hidden attractors [18, 19], and complex transient chaos and hyperchaos [20]. It should be noted that the simplest chaotic circuit has been proposed based on a locally active nonlinear memristive element [4]. Compared to the chaotic circuit shown in [21], the simplest chaotic circuit has following characteristics: (1) the circuit components are connected in a single way, i.e., in series; (2) the number of the circuit components is decreased from four to three; (3) the memristor is locally active.

At a given moment, the resistance of an ideal memristor is represented by the integration of all states before the current moment. This means that the ideal memristor has no memory loss. But the work [22] shows that the width of the doped layer of the $\mathrm{HP} \mathrm{TiO}_{2}$ linear model cannot be equal to zero or the whole width of the model. The $\mathrm{HP} \mathrm{TiO}_{2}$ linear memristor has memory loss. From the definition, the fractional-order derivative depends on the previous history 
of the variable and is not a strictly local operator [23]. The order of the fractional-order derivative is related to the memory loss or the "proximity effect" of some characteristics [12]. Then the nonideal memristor with memory loss mentioned in [22] can be modeled by a fractional-order derivative with the order between 0 and 1 [24]. According to this, there are many memristors modeled with the fractional-order derivative [25-27]. As shown in [24], the fractional-order memristor in the series circuits has capacitive properties or inductive properties by choosing a suitable fractional order; i.e., the fractional order can be regarded as a parameter which is used to control the memory strength and dynamics of the circuit. In [25], the fractional order can be used to control the time period in which the resistance of the memristor increases from the initial value to its maximum. In addition, a noncommensurate fractionalorder autonomous memristor-based circuit is proposed in [27], where the chaotic behavior can be suppressed by applying periodic impulses. In addition, the dynamical system with the locally active equipment can exhibit complexity and emergent behaviors $[28,29]$. Then, it is significant to model the memristor or locally active memristor with the fractional-order derivative and display the dynamics induced by these fractional-order memristors.

The main purpose of this paper is to study the complex dynamical behaviors of a fractional-order system based on a locally active voltage-controlled memristor. By theoretical analyses, the stability conditions of the fractional-order memristive system are listed. The complex dynamical behaviors, such as Hopf bifurcation, period-doubling bifurcation, bistability, and chaos, are displayed numerically. The rest of the paper is organized as follows. In Section 2, the mathematical model of the fractional-order memristor is presented. The fractional-order memristor's fingerprints and local activeness are addressed. In Section 3, an integer-order locally active memristive system is generalized into a fractional-order locally active memristive system. The stability conditions are listed. The complex dynamical behaviors are stated, and numerical simulations are displayed. As an alternative to validating the numerical results, the fractionalorder memristive system is implemented by utilizing Simulink of MATLAB. In Section 4, the effect of the local activeness on complex dynamical behaviors is stated. Section 5 ends with some concluding remarks.

\section{Fractional-Order Locally Active Nonlinear Memristor}

2.1. The Model of the Fractional-Order Memristor. Generally, the memristor can be seen as a sliding resistor whose resistance changes with the charge crossing it. Driven by a bipolar periodic signal, the memristor exhibits a hysteresis loop pinched at the origin in the current-voltage plane. An integer-order nonlinear voltage-controlled memristor is stated as follows [30]:

$$
\left\{\begin{array}{l}
i=W\left(x_{m}\right) v=\left(x_{m}^{2}-x_{m}-1\right) v \\
\dot{x}_{m}=p_{1} x_{m}-p_{2} x_{m}^{3}+p_{4} v-p_{4} x_{m} v
\end{array}\right.
$$

where $v$ and $i$ are the voltage and current of the memristor, respectively, $x_{m}$ is the internal state of the memristor and $W\left(x_{m}\right)=x_{m}^{2}-x_{m}-1$ is the memductance, and $p_{1}, p_{2}, p_{3}$, and $p_{4}$ are the system parameters. By using the trial and error method [30], the parameters are decided as $p_{1}=1.8, p_{2}=3.9$, $p_{3}=1.4$, and $p_{4}=1.5$. Considering the memory effect from the memristor, a fractional-order voltage-controlled memristor $M_{\alpha}$ corresponding to (1) is modeled as follows:

$$
\left\{\begin{array}{l}
i=W_{\alpha}\left(x_{m}\right) v=\left(x_{m}^{2}-x_{m}-1\right) v \\
{ }_{0}^{C} D_{t}^{\alpha} x_{m}=1.8 x_{m}-3.9 x_{m}^{3}+1.4 v-1.5 x_{m} v
\end{array}\right.
$$

where

$$
{ }_{0}^{C} D_{t}^{\alpha} x_{m}(t)=\frac{1}{\Gamma(1-\alpha)} \int_{0}^{t} \frac{\dot{x}_{m}(\tau)}{(t-\tau)^{\alpha}} d \tau, \quad 0<\alpha \leq 1,
$$

is $\alpha$-order derivative of $x_{m}(t)$ in the sense of Caputo's definition given in [23], $\dot{x}_{m}(\tau)$ denotes the first-order derivative of $x_{m}(\tau)$ with respect to $\tau$, and $W_{\alpha}\left(x_{m}\right)=x_{m}^{2}-$ $x_{m}-1$ is the memductance of $\alpha$-order memristor $M_{\alpha}$. The integral process in (3) is the memory process of the memristor. In the circuits, the proposed fractional-order memristor is marked as Figure 1(a).

\subsection{The Characteristics of the Fractional-Order Memristor.} Driven by a sinusoidal voltage source $v(t)=\sin (\omega t)$, hysteresis loops generated in the current-voltage plane are plotted numerically in Figure 2, where $\omega$ is the stimulus frequency. One has the following:

(1) Under different stimulus frequencies or different fractional orders, the hysteresis loops of the fractional-order memristor are pinched at the origin.

(2) The larger the area of the hysteresis loop, the stronger the memory [31]. Let the order $\alpha=0.98$. Figure 2(a) shows that the smaller the stimulus frequency $\omega$, the stronger the memory. As $\omega=1 \mathrm{rad} / \mathrm{s}$, there is another intersection in the hysteresis loop besides the origin and another area $S^{*}$ is displayed. Currently, there are few reports on the new intersection which reflects the nonlinearity of the memristor.

(3) As fixing $\omega=1 \mathrm{rad} / \mathrm{s}$ and decreasing $\alpha$ from 1 to 0 , the area of the hysteresis loop increases; i.e., the strength of the memory increases, referring to Figure 2(b). Simultaneously, the quadrants which the hysteresis loops lie in change from II and IV to II, III, and IV.

2.3. Local Activeness of the Fractional-Order Memristor. A component being capable of providing a power gain is called an active component. If the component provides the power gain within the local range of its variables, the component is locally active.

Based on (2), as $(1-\sqrt{5}) / 2<x_{m}<(1+\sqrt{5}) / 2$, one has $W_{\alpha}\left(x_{m}\right)=x_{m}^{2}-x_{m}-1<0$, the power $p=v \cdot i=W_{\alpha}\left(x_{m}\right)$. $v^{2}<0$, and the memristor can provide the power gain; as $x_{m}<(1-\sqrt{5}) / 2$ or $x_{m}>(1-\sqrt{5}) / 2$, one has $W_{\alpha}\left(x_{m}\right)=$ $x_{m}^{2}-x_{m}-1>0$, the power $p=v \cdot i=W_{\alpha}\left(x_{m}\right) \cdot v^{2}>0$, and 


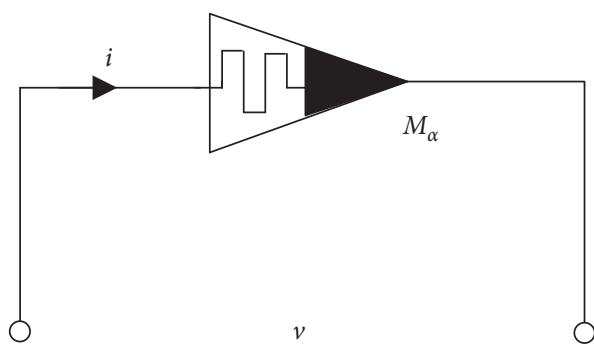

(a)

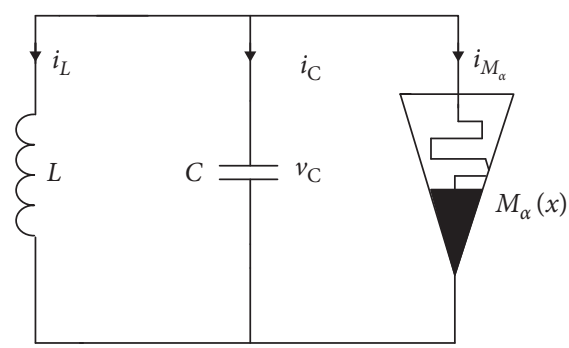

(b)

Figure 1: (a) The fractional-order memristor $M_{\alpha}$; (b) the fractional-order memristive circuit.

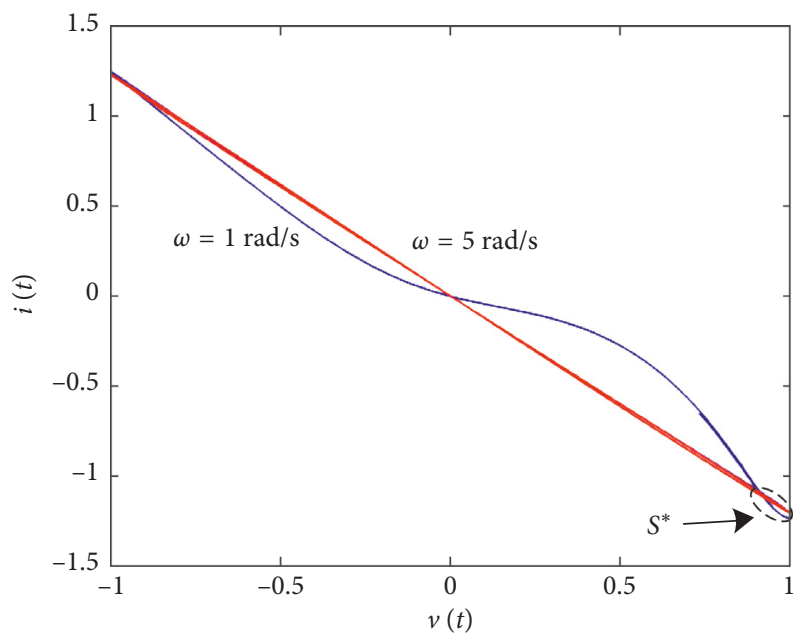

(a)

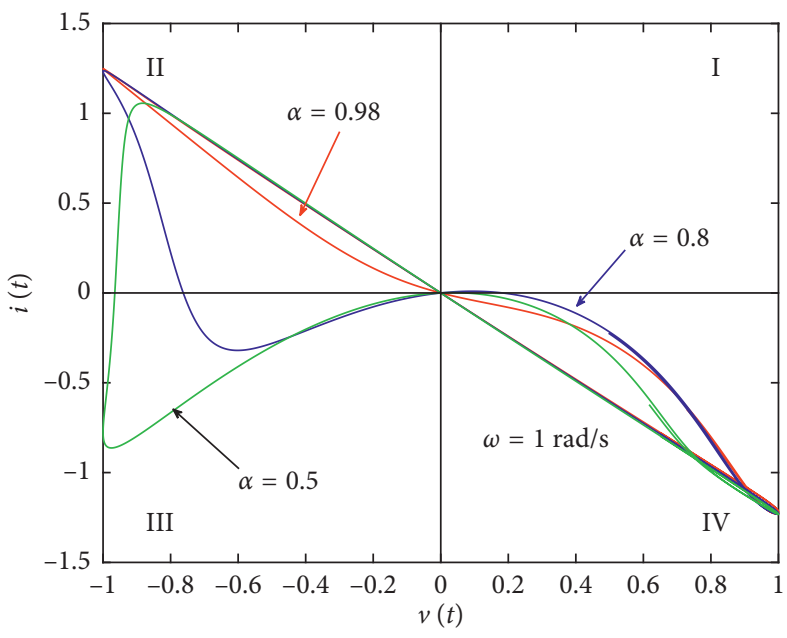

(b)

Figure 2: $v(t)$ versus $i(t)$ of the fractional-order memristor. (a) The order $\alpha=0.98$ with two different frequencies; (b) the frequency $\omega=1 \mathrm{rad} / \mathrm{s}$ with three different fractional orders.

the memristor cannot provide the power gain. So the memristor is locally active.

The above statement implies that the local activeness of the memristor can be decided by the sign of the memductance $W_{\alpha}\left(x_{m}\right)$. Referring to Figure 2, the slope of the hysteresis loop is the memductance. Obviously, the changing of the quadrants of the hysteresis loops changes the sign of the slope or the sign of the memductance $W_{\alpha}\left(x_{m}\right)$. Remembering the characteristics shown in Figure 2(b), it is easy to know that the fractional order has influences on the activeness of the memristor.

\section{Fractional-Order Memristor-Based System}

Besides the memristor can be modeled by the fractionalorder derivative, the capacitor and the inductor can also be modeled by the fractional-order derivative due to the memory effect $[12,32]$. With the fractional-order locally active memristor, a fractional-order memristive circuit is generalized from an integer-order memristive circuit [30], as shown in Figure 1(b), which is modeled by Caputo's fractional-order derivative

$$
\left\{\begin{array}{l}
{ }_{0}^{C} D_{t}^{\alpha} v_{\mathrm{C}}=-a\left[\left(x_{m}^{2}-x_{m}-1\right) v_{\mathrm{C}}+i_{L}\right], \\
{ }_{0}^{C} D_{t}^{\alpha} i_{L}=b v_{\mathrm{C}} \\
{ }_{0}^{C} D_{t}^{\alpha} x_{m}=1.8 x_{m}-3.9 x_{m}^{3}+1.4 v_{\mathrm{C}}-1.5 x_{m} v_{\mathrm{C}}
\end{array}\right.
$$

where $0<\alpha \leq 1, a=1 / C>0, b=1 / L>0$, and $C, L, v_{C}$, and $i_{L}$ are the capacitance, inductance, capacitor voltage, and inductor current, respectively. Letting $\alpha=1$, model (4) is changed into the integer-order model stated in [30].

Denoting $x=v_{\mathrm{C}}, y=i_{L}$, and $z=x_{m}$, (4) is converted into the dimensionless form

$$
\left\{\begin{array}{l}
{ }_{0}^{C} D_{t}^{\alpha} x=-a\left[\left(z^{2}-z-1\right) x+y\right], \\
{ }_{0}^{C} D_{t}^{\alpha} y=b x \\
{ }_{0}^{C} D_{t}^{\alpha} z=1.8 z-3.9 z^{3}+1.4 x-1.5 z x .
\end{array}\right.
$$

For any values of the parameters $a$ and $b$, system (5) has three equilibria: $E_{1}=(0,0,-0.6794), E_{2}=(0,0,0)$, and $E_{3}=(0,0,0.6794)$. For simplicity, the three equilibria are denoted uniformly by $E_{z_{0}}$, where $E_{z_{0}}=\left(0,0, z_{0}\right)$. Thus, $E_{z_{0}}=E_{1}$ as $z_{0}=-0.6794, E_{z_{0}}=E_{2}$ as $z_{0}=0$, and $E_{z_{0}}=E_{3}$ as $z_{0}=0.6794$. 
3.1. The Stability of the Equilibria. The Jacobian matrix of system (5) at $E_{z_{0}}$ is

$$
J_{E}=\left(\begin{array}{ccc}
-a\left(z_{0}^{2}-z_{0}-1\right) & -a & 0 \\
b & 0 & 0 \\
1.4-1.5 z_{0} & 0 & 1.8-11.7 z_{0}^{2}
\end{array}\right) \text {. }
$$

The characteristic polynomial equation of system (5) at $E_{z_{0}}$ is yielded as

$$
\left(\lambda-1.8+11.7 z_{0}^{2}\right)\left[\lambda^{2}+a\left(z_{0}^{2}-z_{0}-1\right) \lambda+a b\right]=0,
$$

which indicates that roots of (7) depend on the three equilibria. The roots of (7) are called the eigenvalues of Jacobian matrix $J_{E}$. The following lemma is needed.

Lemma 1 [33]. The fractional-order nonlinear system

$$
{ }_{0}^{C} D_{t}^{\alpha} \mathbf{X}=\mathbf{f}(\mathbf{X}), \quad 0<\alpha \leq 1,
$$

is asymptotically stable at the equilibrium $E=\left(x_{0}, y_{0}, z_{0}\right)$ if all eigenvalues $\lambda$ of Jacobian matrix $J_{E}$ satisfy the condition

$$
|\arg (\lambda)|>\frac{\alpha \pi}{2}
$$

where $\quad \mathbf{X}=(x, y, z)^{T}, \quad \mathbf{f}(\mathbf{X})=\left[f_{1}(\mathbf{X}), f_{2}(\mathbf{X}), f_{3}(\mathbf{X})\right]^{T}$, $f_{i}(\mathbf{X})=f_{i}(x, y, z)(i=1,2,3)$, and $\arg (\lambda)$ is the principal argument of the eigenvalue $\lambda$.

Obviously, equation (7) has a real root $\lambda_{1}=1.8-11.7 z_{0}^{2}$. Considering the sign of $\lambda_{1}$, two cases are discussed hereinafter.

Case 1. $\lambda_{1}$ is positive

If $\lambda_{1}>0$, one has $\left|z_{0}\right|<0.3922$ and the equilibrium $E_{z_{0}}$ is unstable. Besides the positive root $\lambda_{1}$, equation (7) has another two roots:

$$
\lambda_{2,3}=\frac{-a\left(z_{0}^{2}-z_{0}-1\right) \pm \sqrt{a^{2}\left(z_{0}^{2}-z_{0}-1\right)^{2}-4 a b}}{2} .
$$

It is easy to know that $-0.454<z_{0}^{2}-z_{0}-1<-1.2384$ as $\left|z_{0}\right|<0.3922$. Two cases are listed:

(1) $\lambda_{2}$ and $\lambda_{3}$ are the real roots and $\left(z_{0}^{2}-z_{0}-1\right)^{2} \geq 4 b / a$ holds. Based on (10), one has $\lambda_{1}>0, \lambda_{2}>0$, and $\lambda_{3}>0$.

(2) $\lambda_{2}$ and $\lambda_{3}$ are the complex roots; i.e., $\left(z_{0}^{2}-z_{0}-1\right)^{2}$ $<4 b / a$ holds. The real parts of the conjugate complex roots are $\operatorname{Re}\left(\lambda_{2,3}\right)=-a\left(z_{0}^{2}-z_{0}-1\right) / 2>0$.

Case 2. $\lambda_{1}$ is negative

As $\lambda_{1}=1.8-11.7 z_{0}^{2}<0$, one has $\left|z_{0}\right|>0.3922$. Similar to Case 1, two cases are stated as follows:

(1) $\lambda_{2}$ and $\lambda_{3}$ are the real roots. Then $\left(z_{0}^{2}-z_{0}-1\right)^{2} \geq 4 b / a$ holds and

$$
\operatorname{sgn}\left(\lambda_{2}\right)=\operatorname{sgn}\left(\lambda_{3}\right)=\operatorname{sgn}\left(-a\left(z_{0}^{2}-z_{0}-1\right)\right),
$$

where $\operatorname{sgn}(\cdot)$ is the symbolic function. Due to

$$
g\left(z_{0}\right)=z_{0}^{2}-z_{0}-1=\left[z_{0}-\frac{1-\sqrt{5}}{2}\right]\left[z_{0}-\frac{1+\sqrt{5}}{2}\right]
$$

one has

$$
-a\left(z_{0}^{2}-z_{0}-1\right) \begin{cases}<0, & z_{0}<-0.618 \text { or } z_{0}>1.618 \\ >0, & -0.618<z_{0}<-0.3299 \text { or } 0.3299<z_{0}<1.618 .\end{cases}
$$

where $(1-\sqrt{5}) / 2 \approx-0.618$ and $(1+\sqrt{5}) / 2 \approx$ 1.618. The inequalities $-0.618<z_{0}<-0.3299$ and $z_{0}>1.618$ can be neglected because $z_{0}$ of the three equilibria is not in these regions. As $z_{0}<-0.618$, one has $\lambda_{2}<0$ and $\lambda_{3}<0$. As $0.3229<z_{0}<1.618$, one has $\lambda_{2}>0$ and $\lambda_{3}>0$.

(2) $\lambda_{2}$ and $\lambda_{3}$ are the complex roots. The inequality $\left(z_{0}^{2}-z_{0}-1\right)^{2}<4 b / a$ holds. As $z_{0}<-0.618, \lambda_{2}$ and $\lambda_{3}$ are the conjugate complex roots with negative real parts $\operatorname{Re}\left(\lambda_{2,3}\right)=-a\left(z_{0}^{2}-z_{0}-1\right) / 2<0$. As $0.3229<$ $z_{0}<1.618, \lambda_{2}$ and $\lambda_{3}$ are the conjugate complex roots with positive real parts $\operatorname{Re}\left(\lambda_{2,3}\right)=-a\left(z_{0}^{2}-z_{0}-1\right)$ $/ 2>0$.

The above discussion can be concluded in Tables 1 and 2 . Tables 1 and 2 show that $E_{1}=(0,0,-0.6794)$ is stable and $E_{2}=(0,0,0)$ is unstable for any $a>0, b>0$, and any order $\alpha \in(0,1]$. But for $E_{3}=(0,0,0.6794)$, two cases are stated:

(1) if $1.4831 \geq 4 b / a, E_{3}$ is unstable for any order $\alpha$ because $\lambda_{2}>0$ and $\lambda_{3}>0$;

(2) if $1.4831<4 b / a$, there are two conjugated complex roots at $E_{3}$ as

$$
\lambda_{2,3}=\operatorname{Re}\left(\lambda_{2,3}\right) \pm j \operatorname{Im}\left(\lambda_{2,3}\right)=\frac{-a\left(z_{0}^{2}-z_{0}-1\right) \pm j \sqrt{4 a b-a^{2}\left(z_{0}^{2}-z_{0}-1\right)^{2}}}{2}
$$


TABLE 1: The eigenvalues as $\left(z_{0}^{2}-z_{0}-1\right)^{2} \geq 4 b / a$.

\begin{tabular}{lccc}
\hline$z_{0}$ & $(-\infty,-0.618)$ & $(-0.3299,0.3299)$ & $(0.3299,1.618)$ \\
\hline$\lambda_{1}$ & $\lambda_{1}<0$ & $\lambda_{1}>0$ & $\lambda_{1}<0$ \\
$\lambda_{2}$ & $\lambda_{2}<0$ & $\lambda_{2}>0$ & $\lambda_{2}>0$ \\
$\lambda_{3}$ & $\lambda_{3}<0$ & $\lambda_{3}>0$ & $\lambda_{3}>0$ \\
Equilibrium & $E_{1}$ & $E_{2}$ & $E_{3}$
\end{tabular}

TABLe 2: The eigenvalues as $\left(z_{0}^{2}-z_{0}-1\right)^{2}<4 b / a$.

\begin{tabular}{lccc}
\hline$z_{0}$ & $(-\infty,-0.618)$ & $(-0.3299,0.3299)$ & $(0.3299,1.618)$ \\
\hline$\lambda_{1}$ & $\lambda_{1}<0$ & $\lambda_{1}>0$ & $\lambda_{1}<0$ \\
$\lambda_{2}$ & $\operatorname{Re}\left(\lambda_{2}\right)<0$ & $\operatorname{Re}\left(\lambda_{2}\right)>0$ & $\operatorname{Re}\left(\lambda_{2}\right)>0$ \\
$\lambda_{3}$ & $\operatorname{Re}\left(\lambda_{3}\right)<0$ & $\operatorname{Re}\left(\lambda_{3}\right)>0$ & $\operatorname{Re}\left(\lambda_{3}\right)>0$ \\
Equilibrium & $E_{1}$ & $E_{2}$ & $E_{3}$ \\
\hline
\end{tabular}

where $j^{2}=-1$. If $\left|\arg \left(\lambda_{2,3}\right)\right|>(\alpha \pi) / 2$ holds, one has

$$
\frac{\sqrt{4 a b-1.4831 a^{2}}}{1.2178 a}>\tan \frac{\alpha \pi}{2} \text {. }
$$

By Lemma $1, E_{3}=(0,0,0.6794)$ is stable as $\sqrt{4 a b-1.4831 a^{2}} /(1.2178 a)>\tan (\alpha \pi / 2)$ holds; $E_{3}$ is unstable as $\sqrt{4 a b-1.4831 a^{2}} /(1.2178 a)>\tan (\alpha \pi / 2)$ does not hold.

Based on the above discussion, the following theorem is established.

Theorem 1. For system (5) $(\alpha \in(0,1])$, the stabilities of three equilibria $E_{1}, E_{2}$, and $E_{3}$ are as follows:

(1) Equilibrium $E_{1}=(0,0,-0.6794)$ is stable for any $a>0$, $b>0$ and any order $\alpha \in(0,1]$;

(2) Equilibrium $E_{2}=(0,0,0)$ is unstable for any $a>0$, $b>0$ and any order $\alpha \in(0,1]$;

(3) As $1.4831 \geq 4 b / a, E_{3}$ is unstable for any order $\alpha \in(0,1] ; \quad$ as $1.4831<4 b / a, \quad E_{3}$ is stable if $\sqrt{4 a b-1.4831 a^{2}} /(1.2178 a)>\tan (\alpha \pi / 2)$, and $E_{3}$ is unstable if $\sqrt{4 a b-1.4831 a^{2}} /(1.2178 a)<\tan (\alpha \pi / 2)$.

Remark 1. For a fractional-order system, at a parameter $\varepsilon=\varepsilon_{0}$, a pair of conjugated complex eigenvalues $\lambda_{1,2}$ satisfy $\left|\arg \left(\lambda_{1,2}\right)\right|=(\alpha \pi) / 2$ and other eigenvalues are in stable zones. While the parameter $\varepsilon>\varepsilon_{0},\left|\arg \left(\lambda_{1,2}\right)\right|<(\alpha \pi) / 2$, Hopf bifurcation is generated at $\varepsilon=\varepsilon_{0}$ [34].

For system (5), based on Table 2 , at $E_{3}=(0,0,0.6794)$, as $1.4831<4 b / a$ holds, one has

(1) $\lambda_{1}<0$;

(2) $\left|\arg \left(\lambda_{2,3}\right)\right|>(\alpha \pi) / 2 \quad$ as $\quad \alpha<(2 / \pi) \arctan$ $\left(\sqrt{4 a b-1.4831 a^{2}} / 1.2178 a\right)$

(3) $\left(\arg \left(\lambda_{2,3}\right)\right)<(\alpha \pi) / 2 \quad$ as $\quad \alpha>(2 / \pi) \arctan$ $\left(\sqrt{4 a b-1.4831 a^{2}} / 1.2178 a\right)$. This means that Hopf bifurcation is generated as the order $\alpha=(2 / \pi) \arctan \left(\sqrt{4 a b-1.4831 a^{2}} / 1.2178 a\right)$ at the equilibrium $E_{3}$.

Remark 2. For simplicity, the eigenvalues $\left(\lambda_{1}, \lambda_{2}, \lambda_{3}\right)$ are denoted as $(\gamma, \sigma+j \omega, \sigma-j \omega)$, where $\gamma, \sigma$, and $\omega$ are all real numbers. A saddle-focus point is called a saddle-focus point of index 1 if $\gamma>0$ and $\sigma<0$, and a saddle-focus point is called a saddle-focus point of index 2 if $\gamma<0$ and $\sigma>0$ [35]. As pointed out in [35], the saddle-focus points of index 2 are crucial to the generation of chaotic attractors. Usually, in chaotic systems, scrolls are generated around the saddlefocus points of index 2, and the saddle-focus points of index 1 are responsible only for connecting the scrolls.

3.2. Numerical Illustrations. For better comparisons with the integer-order memristive circuit systems, in this section, the parameter is chosen as $a=10 / 3$ [30].

Case 1. $a=10 / 3$, and $b=10$.

In this case, $a=10 / 3(C=300 \mathrm{mF})$ and $b=10(L=100$ $\mathrm{mH}$ ) satisfy $4 b / a=12>1.4831$. To make $E_{3}$ stable, by Theorem 1, the order $\alpha$ is satisfied as

$$
\begin{aligned}
\alpha & <\left(\frac{2}{\pi}\right) \arctan \left(\frac{\sqrt{4 a b-1.4831 a^{2}}}{1.2178 a}\right) \\
& =\left(\frac{2}{\pi}\right) \arctan \left(\frac{\sqrt{4 \cdot(10 / 3) \cdot 10-1.4831 \cdot(10 / 3)^{2}}}{1.2178 \cdot(10 / 3)}\right) \\
& =0.7713 .
\end{aligned}
$$

Then equilibrium $E_{3}=(0,0,0.6794)$ is stable as the order $\alpha<0.7713$, and $E_{3}$ is unstable as the order $\alpha>0.7713$. By Remark 1, at equilibrium $E_{3}$, Hopf bifurcation is generated as $\alpha=0.7713$.

At the equilibrium $E_{2}$, the two complex eigenvalues are

$$
\begin{aligned}
\lambda_{2,3} & =\frac{-a\left(z_{0}^{2}-z_{0}-1\right) \pm j \sqrt{4 a b-a^{2}\left(z_{0}^{2}-z_{0}-1\right)^{2}}}{2} \\
& =\frac{5 \pm 5 \sqrt{11} j}{2}, \quad\left(j^{2}=-1\right) .
\end{aligned}
$$

If $\tan \left(\arg \left(\lambda_{2,3}\right)\right)=\sqrt{11}>\tan (\alpha \pi / 2)$, one has $\alpha<0.8136$. This means that the two complex eigenvalues $\lambda_{2,3}$ of the equilibrium $E_{2}$ lie in the stable zone. Due to $\lambda_{1}>0$ and Remark 2, $E_{2}$ is an unstable saddle-focus with index 1 and $E_{3}$ is an unstable saddle-focus with index 2. While $\alpha>0.8136$, the two complex eigenvalues $\lambda_{2,3}$ of the equilibrium $E_{2}$ lie in the unstable zone, $E_{2}$ is an unstable node-focus, and $E_{3}$ is an unstable saddle-focus with index 2 . The types of three equilibria are listed in Table 3. One has the following:

(1) As $0<\alpha<0.7713$, there are two steady states of $E_{1}$ and $E_{3}$.

(2) As $0.7713<\alpha<0.8136$, there are two steady states of $E_{1}$ and the limit cycle bifurcated from unstable saddle-focus $E_{3}$.

(3) As $0.8136<\alpha<1$, the stability of $E_{1}$ is unchanged. $E_{2}$ is changed from unstable saddle-focus into unstable node-focus because the two complex roots $\lambda_{2,3}$ of $E_{2}$ enter into the unstable zone. 
TABLE 3: The types of the equilibria.

\begin{tabular}{lccc}
\hline$\alpha$ & $E_{1}$ & $E_{2}$ & $E_{3}$ \\
\hline $0,0.7713)$ & Stable & $\begin{array}{c}\text { Unstable saddle- } \\
\text { focus }\end{array}$ & Stable \\
0.7713 & Stable & $\begin{array}{c}\text { Unstable saddle- } \\
\text { focus }\end{array}$ & $\begin{array}{c}\text { Hopf bifurcation } \\
\text { point }\end{array}$ \\
$(0.7713,0.8136)$ & Stable & $\begin{array}{c}\text { Unstable saddle- } \\
\text { focus }\end{array}$ & $\begin{array}{c}\text { Unstable saddle- } \\
\text { focus }\end{array}$ \\
0.8136 & Stable & Unstable point & $\begin{array}{c}\text { Unstable saddle- } \\
\text { focus }\end{array}$ \\
$(0.8136,1)$ & Stable & $\begin{array}{c}\text { Unstable node- } \\
\text { focus }\end{array}$ & $\begin{array}{c}\text { Unstable saddle- } \\
\text { focus }\end{array}$ \\
\hline
\end{tabular}

Remark 3. It is found that $E_{3}$ is an unstable saddle-focus with index 2 as the order $\alpha>0.7713$. Due to Remark 2, for system (5), chaotic attractors may be generated as the order $\alpha>0.7713$.

Fix the initial values $(0.1,0.1,0.2)$. Figure $3(a)$ is the bifurcation diagram of the local maxima of the variable $z$ about the order $\alpha$, which shows that Hopf bifurcation is generated at $\alpha=0.7713$. As $0.7713<\alpha<0.8136$, system (5) displays a limit cycle bifurcated from the equilibrium $E_{3}$. As $0.8136<\alpha<0.84$, the limit cycle induced by Hopf bifurcation disappears and the phase portrait limits to the stable point $E_{1}$. Increasing $\alpha$ from 0.84 to 1 , the period-doubling bifurcation occurs in system (5). Figure 3(b) is the first two Lyapunov exponents of system (5) according to the MATLAB code of [36]. As $\alpha>0.97$, the first Lyapunov exponent $\mathrm{LE}_{1}>0$ and system (5) enters into the chaos.

Case 2. $\alpha=0.99$, and $a=10 / 3$.

In this case, if the inequality $4 b / a>1.4831$ holds, one has $b>1.2359$. Same as the integer-order case [30], the parameter $L$ is set in $[70 \mathrm{mH} ; 100 \mathrm{mH}$; i.e., the parameter $b$ is in $[10,100 / 7]$. If $E_{3}$ is stable, by Theorem 1 , the parameter $b$ should be satisfied as

$$
\begin{aligned}
b & >\frac{\left[1.2178^{2} \cdot a \cdot \tan ^{2}(\alpha \pi / 2)+1.4831 a\right]}{4} \\
& =\frac{\left[1.2178^{2} \cdot(10 / 3) \cdot \tan ^{2}(0.99 \pi / 2)+1.4831(10 / 3)\right]}{4} \\
& =5009.2 .
\end{aligned}
$$

This means that $E_{3}$ is a saddle-focus with index 2 for any $b \in[10,100 / 7]$ or any $L \in[70 \mathrm{mH}, 100 \mathrm{mH}]$. Thus, chaotic attractors may be generated at $E_{3}$ as $L$ increases from $70 \mathrm{mH}$ to $100 \mathrm{mH}$.

At the equilibrium $E_{2}$, two complex eigenvalues are

$$
\begin{aligned}
\lambda_{2,3} & =\frac{-a\left(z_{0}^{2}-z_{0}-1\right) \pm j \sqrt{4 a b-a^{2}\left(z_{0}^{2}-z_{0}-1\right)^{2}}}{2} \\
& =\frac{(10 / 3) \pm j \sqrt{4(10 / 3) b-(10 / 3)^{2}}}{2} .
\end{aligned}
$$

If $\tan \left(\arg \left(\lambda_{2,3}\right)\right)=\sqrt{120 b-100} / 10>\tan (0.99 \pi / 2)$, one has $b>3377.65$ or $L<0.2961 \mathrm{mH}$. This means that two complex eigenvalues $\lambda_{2,3}$ of the equilibrium $E_{2}$ lie in the unstable zone as $L \in\left[70 \mathrm{mH}, 100 \mathrm{mH}\right.$. Due to $\lambda_{1}>0, E_{2}$ is an unstable node-focus which is that same as the former case of $0.8136<\alpha<1$.

The bifurcation diagram of the variable $z$ about the parameter $L$ is plotted numerically in Figure 3(c). The first two Lyapunov exponents are shown in Figure 3(d). It is found that system (5) goes into chaos by the period-doubling bifurcation. After system (5) enters into chaos, there suddenly appears several periodic windows (PWs) as $L>86 \mathrm{mH}$. Compared to the integer-order model in [30], the fractionalorder memristive system described by system (5) has more periodic windows. The minimum of the parameter $L$ for system (5) entering into chaos is larger than the minimum of the parameter $L$ shown in [30].

Therefore, the generated complicated dynamical behaviors of system (5) are related to the coexistence of the stable point $E_{1}$, the unstable node-focus $E_{2}$, and the saddlefocus $E_{3}$.

3.3. Bistability Behaviors. Fixing the parameter values and choosing different initial values, a nonlinear system shows two steady states. This behavior is called the bistability behavior. The bistability behavior reflects the sensitivity of the system to its initial values. For different order $\alpha$ or different inductance $L$, different bistability behaviors appear in system (5), which is listed in Tables 4 and 5.

Bistability behaviors for different fractional orders are plotted numerically in Figure 4. Figure 4(a) is two steady states of the stable points $E_{1}$ and $E_{3}$, Figure $4(\mathrm{~b})$ is two steady states of the stable point $E_{1}$ and period-2 cycle, Figure $4(\mathrm{c})$ is two steady states of the stable point $E_{1}$ and period- 4 cycle, and Figure $4(\mathrm{~d})$ is two steady states of the stable point $E_{1}$ and chaotic attractor. Compared to the bistability of stable point and chaotic attractor in the integer-order model, a conclusion that the fractional-order derivative can enrich the bistability behaviors is drawn.

Furthermore, the attraction basins in the $x(0)-z(0)$ plane are used to validate the bistability behaviors of system (5) for four different orders $\alpha$, as shown in Figure 5, where $y(0)=0.1$. The light blue, green, yellow, and magenta regions represent the initial value regions for generating period-2, period-4, chaotic, and stable point behaviors, respectively.

3.4. Block Designs of System (5) in Simulink of MATLAB. By utilizing Simulink of MATLAB, the fractional-order system (5) can be implemented to confirm the above numerical plots.

Figure 6 is the block diagrams in Simulink of MATLAB. Figure $6(\mathrm{a})$ is the $\alpha$-order differentiator block design. The top in Figure 6(a) is the masked block of the $\alpha$-order derivative and the bottom in Figure 6(a) is the filter in fractional-order differentiator (here the Oustaloup recursive filter is used). The masking technique of fo_diff.mdl is provided in [37]. Double clicking the block of Fractional Der $s^{\alpha}$, the order $\alpha$ can be changed by the parameter dialog box. Furthermore, if 


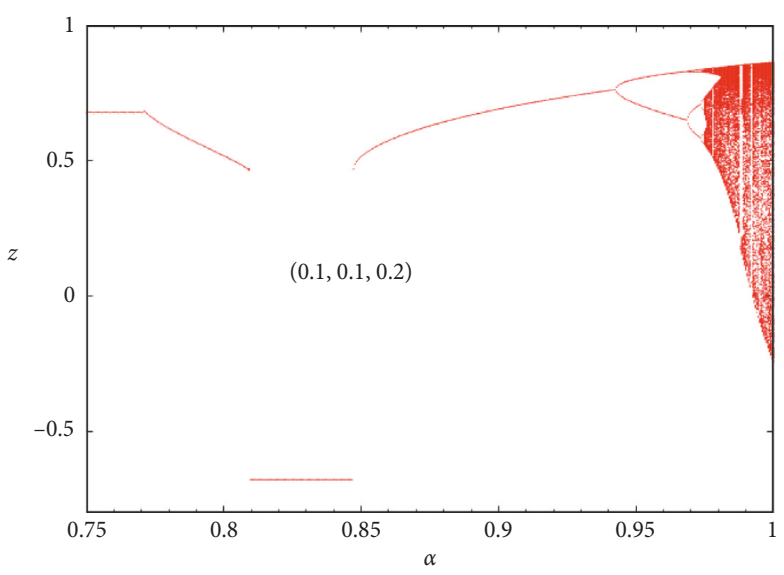

(a)

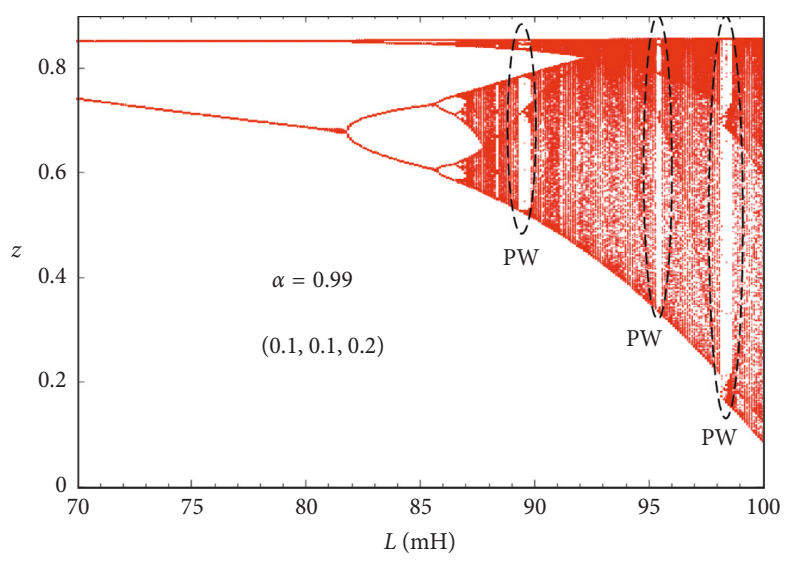

(c)

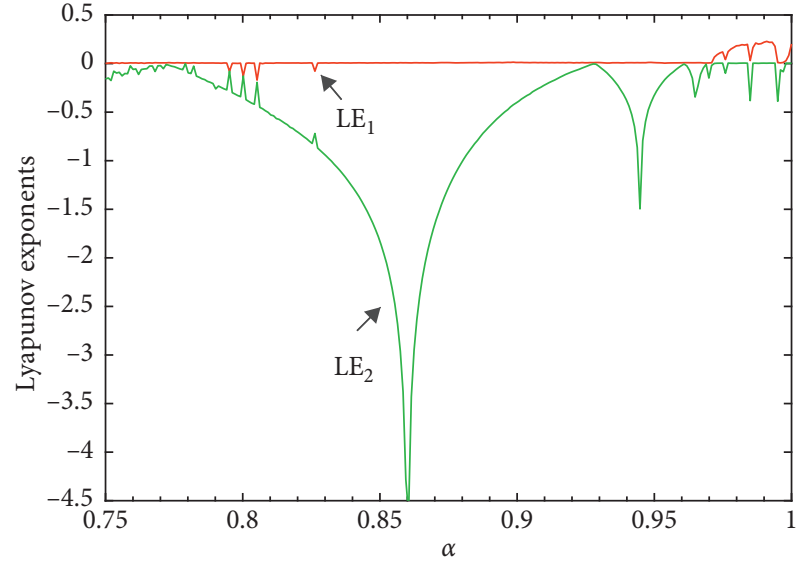

(b)

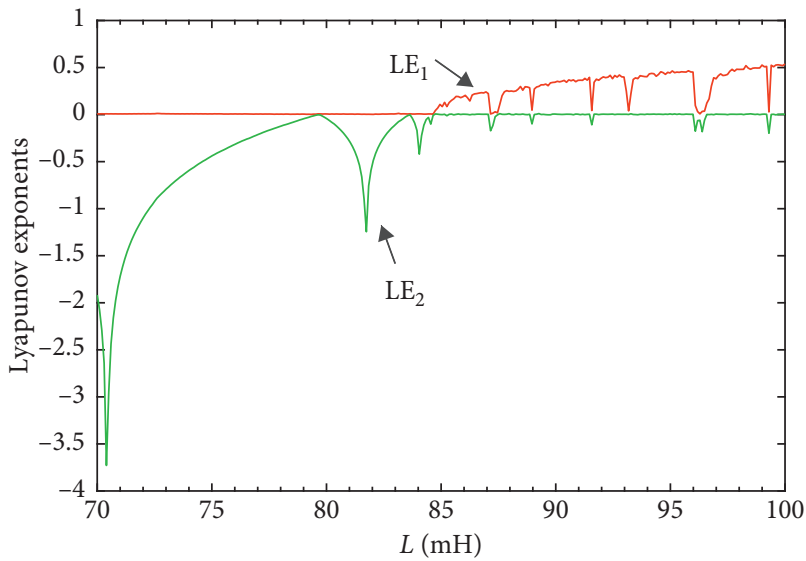

(d)

Figure 3: Numerical simulations of system (5), $a=10 / 3, b=10$, and the initial values $(0.1,0.1,0.2)$. (a) Bifurcation diagram of the local maxima of the variable $z$ about the order $\alpha$; (b) first two Lyapunov exponents of Case 1; (c) bifurcation diagram of the local maxima of the variable $z$ about the parameter $L$; (d) first two Lyapunov exponents of Case 2.

TABLE 4: Bistability in the case of $a=10 / 3$ and $b=10$.

\begin{tabular}{lc}
\hline$\alpha$ & Two steady states \\
\hline$(0,0.7713)$ & Two stable points of $E_{1}$ and $E_{2}$ \\
$(0.7713,0.84)$ & Stable point $E_{1}$ and limit cycle \\
$(0.84,0.97)$ & Stable point $E_{1}$ and limit cycle \\
$(0.97,1)$ & Stable point $E_{1}$ and chaotic attractor \\
\hline
\end{tabular}

$$
{ }_{0}^{C} D_{t}^{\alpha} x(t)=f(t), \quad 0<\alpha<1,
$$

one has [38]

$$
{ }_{0}^{C} D_{t}^{1-\alpha}\left({ }_{0}^{C} D_{t}^{\alpha} x(t)\right)=\frac{\mathrm{d} x(t)}{\mathrm{d} t}
$$

or

$$
\frac{\mathrm{d} x(t)}{\mathrm{d} t}={ }_{0}^{C} D_{t}^{1-\alpha} f(t)
$$

Then in the block diagram of the fractional-order memristor or in Figure 6(b), the $\alpha$-order fractional-order derivative of the state is obtained by an integrator.mdl and the fo_diff.mdl of Fractional Der $s^{(1-\alpha)}$.
TABLE 5: Bistability in the case of $\alpha=0.99$ and $a=10 / 3$.

\begin{tabular}{lc}
\hline$L \mathrm{mH}$ & Two steady states \\
\hline$(70,86)$ & Stable point $E_{1}$ and limit cycle \\
$(86,100)$ & Stable point $E_{1}$ and chaotic attractor \\
\hline
\end{tabular}

The input voltage in Figure 6(b) is $v(t)=\sin (\omega t)$. Fixing the order $\alpha=0.98$, the hysteresis loops of different input frequency $\omega$ obtained in the scope ( $X Y$ graph) are shown in Figure $7(\mathrm{a})$. Fixing the input frequency $\omega=1 \mathrm{rad} / \mathrm{s}$, the hysteresis loops of different order $\alpha$ obtained in the scope (XY graph) are shown in Figure $7(\mathrm{~b})$. Figure 7 plotted in Simulink of MATALB is consistent with Figure 2.

The block diagram of system (5) in Simulink of MATLAB is designed in Figure 8. By using (22), the $\alpha$-order fractional-order derivative of the state is obtained by an integrator.mdl and the fo_diff.mdl of Fractional Der $s^{(1-\alpha)}$. The initial values of the states are set in three integrators. Setting $1-\alpha=0.25$ in Figure 8 , bistability behaviors of $\alpha=0.75$ obtained in the scope ( $X Z$ graph) are shown in Figure $9(\mathrm{a})$. Setting $1-\alpha=0.01$ in Figure 8 , bistability behaviors of $\alpha=0.99$ obtained in the scope ( $X Z$ graph) are shown in Figure 9(b). 


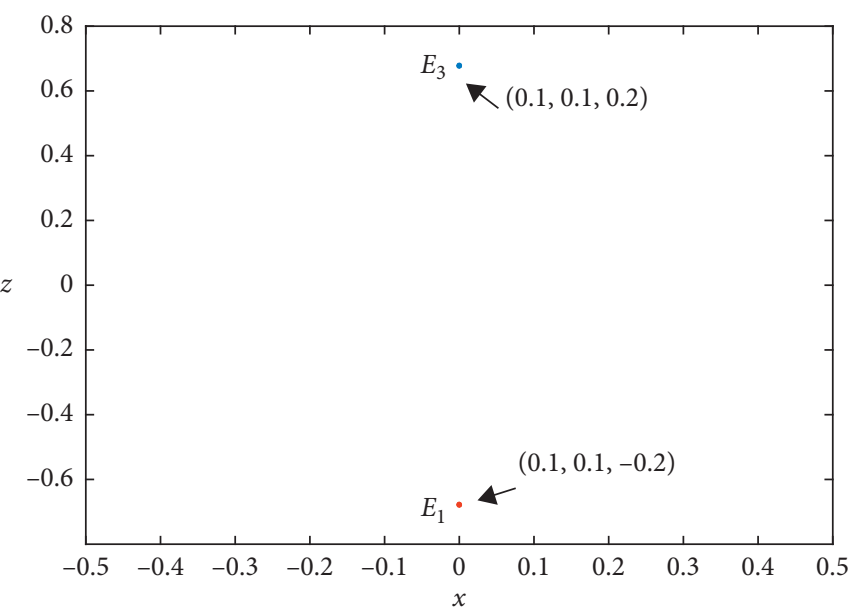

(a)

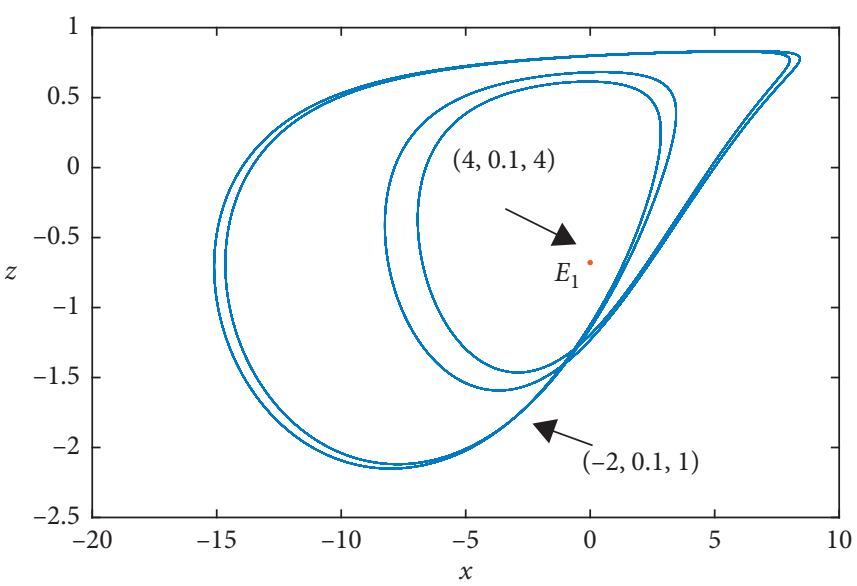

(c)

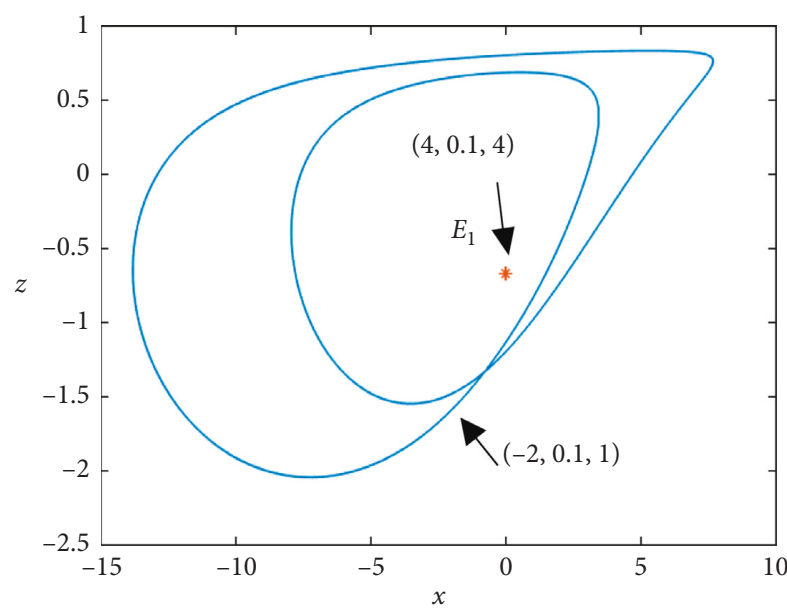

(b)

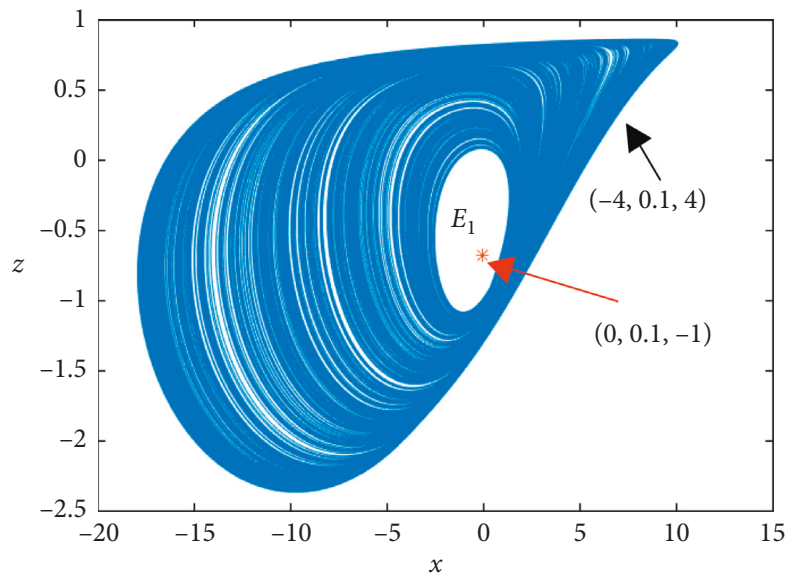

(d)

Figure 4: Bistability behaviors with different fractional orders. (a) $\alpha=0.75$, two sets of the initial values $(0.1,0.1,0.2)$ and $(0.1,0.1,-0.2)$; (b) $\alpha=0.96$ and two sets of the initial values $(4,0.1,4)$ and $(-2,0.1,1)$; (c) $\alpha=0.97$ and two sets of the initial values $(4,0.1,4)$ and $(-2,0.1,1)$; (d) $\alpha=0.99$ and two sets of the initial values $(-4,0.1,4)$ and $(0,0.1,-1)$.

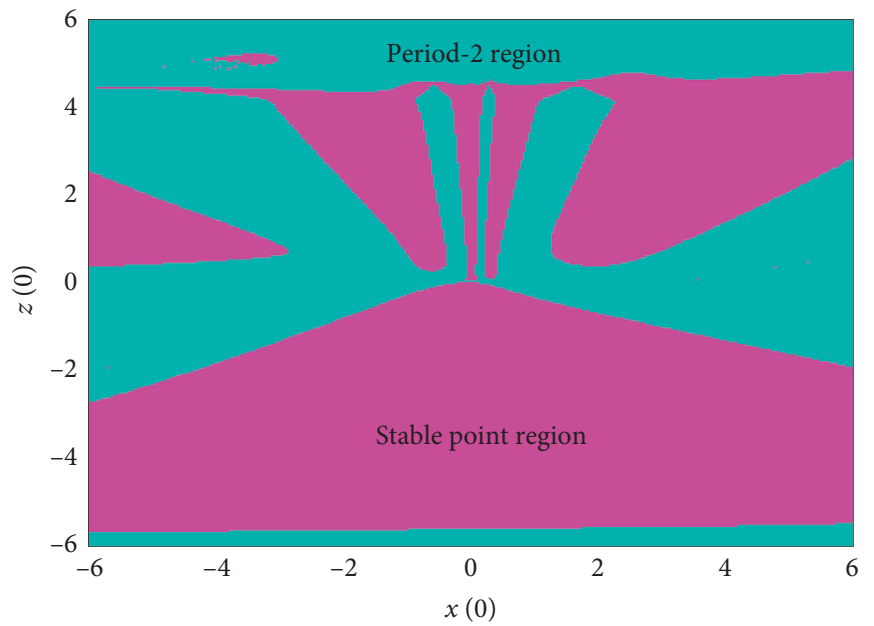

(a)

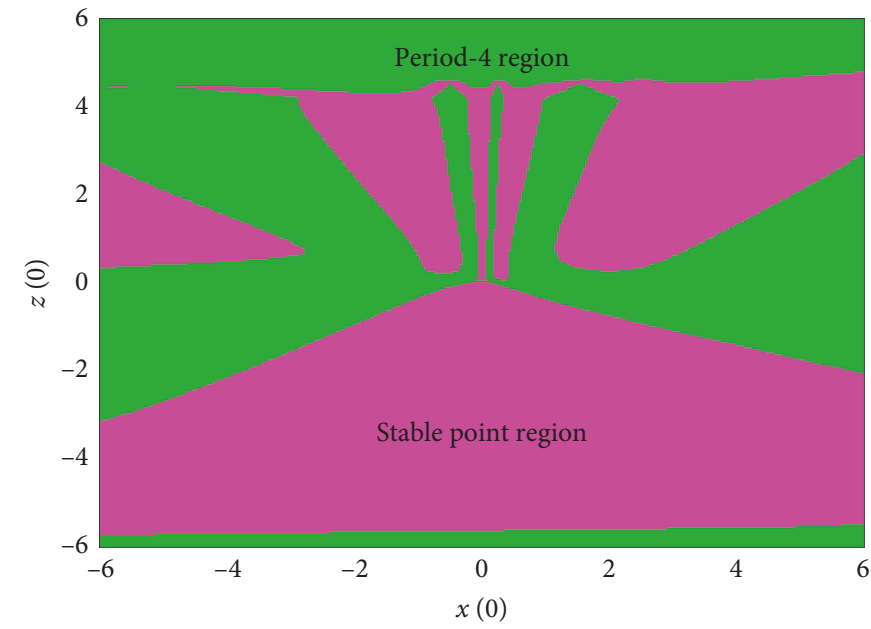

(b)

Figure 5: Continued. 


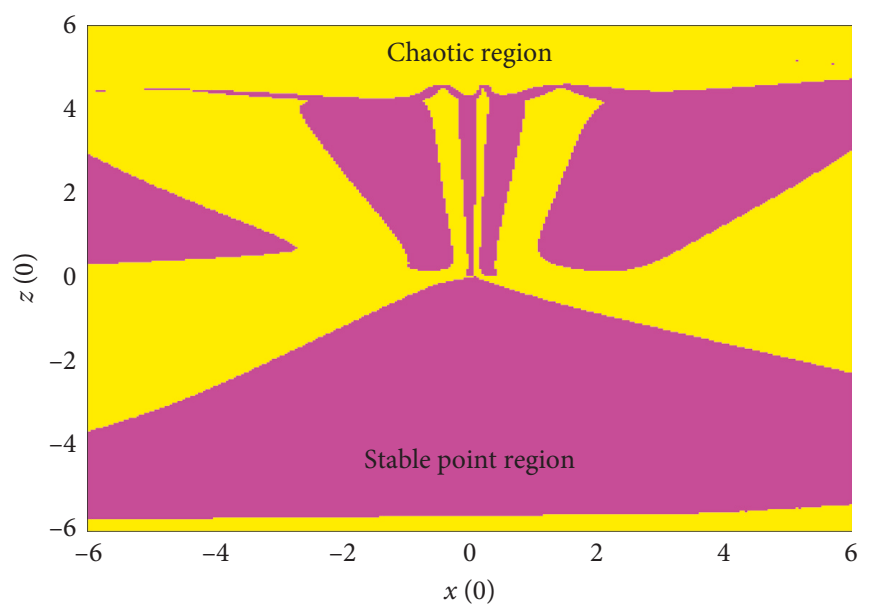

(c)

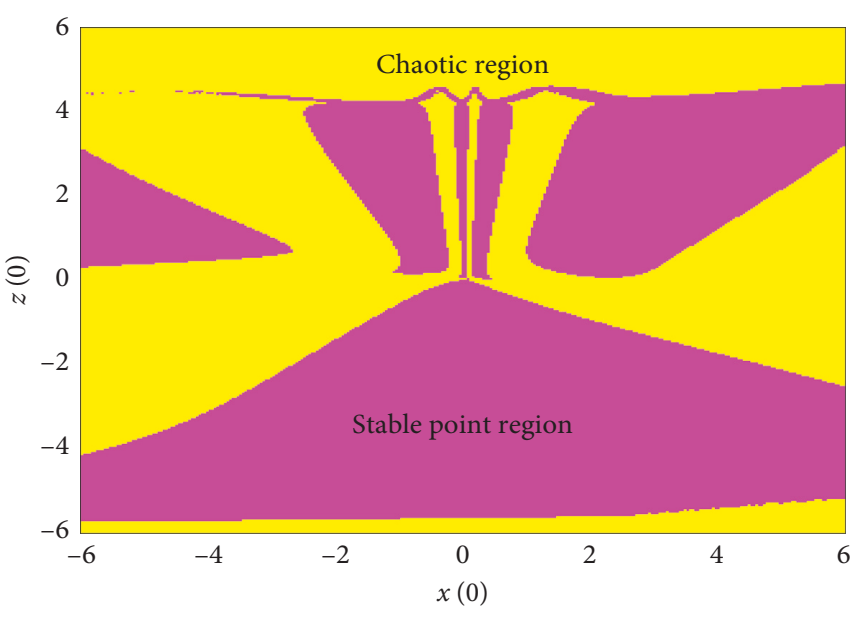

(d)

FIGURE 5: Bistability behaviors demonstrated by the attraction basins in the $x(0)-y(0)$ plane for four different fractional orders with $y(0)=0.1$. (a) Attraction basin for $\alpha=0.96$; (b) attraction basin for $\alpha=0.97$; (c) attraction basin for $\alpha=0.98$; (d) attraction basin for $\alpha=0.99$.

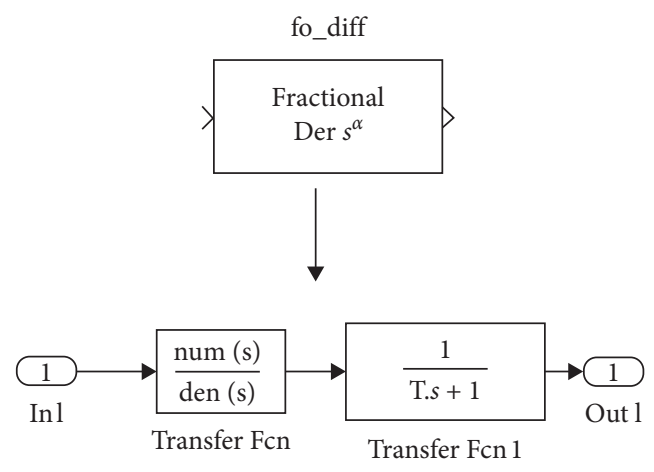

(a)

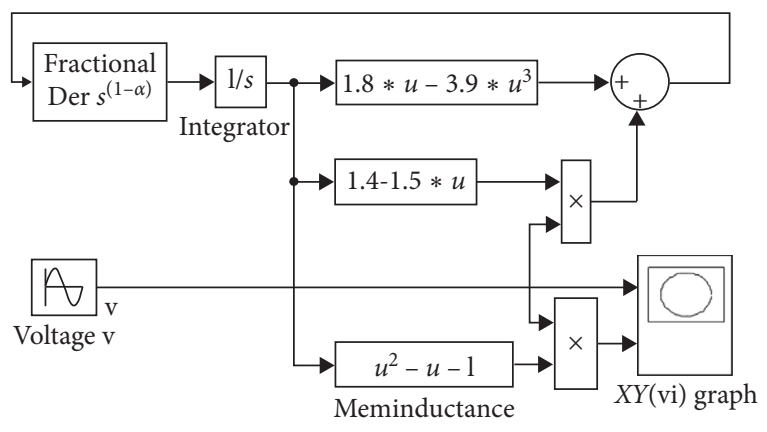

(b)

Figure 6: Block diagrams in Simulink of MATLAB. (a) Differentiator block design 4 of the order $\alpha$; (b) block diagram of the fractional-order memristor.

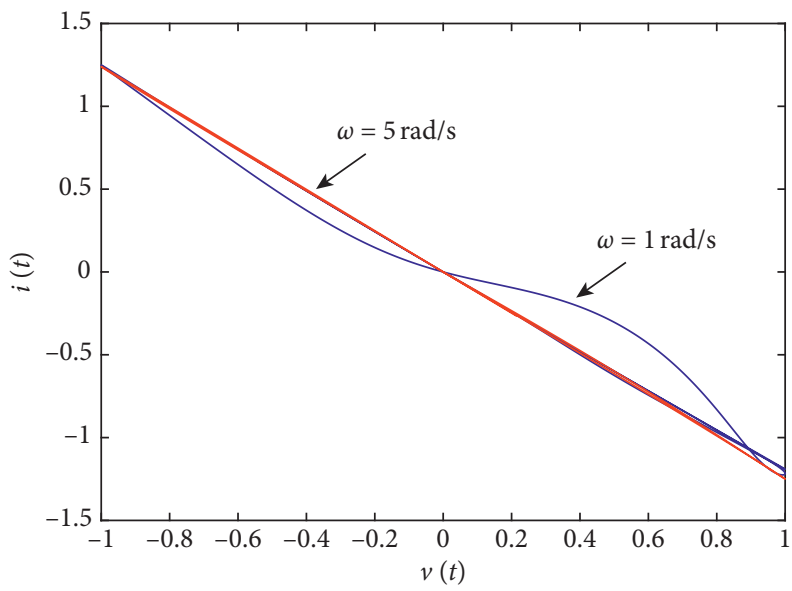

(a)

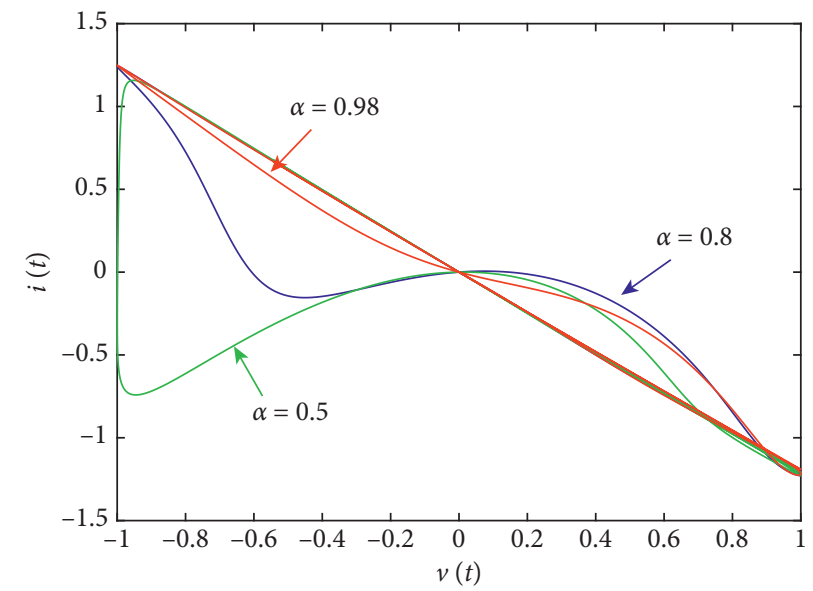

(b)

Figure 7: $v(t)$ versus $i(t)$ of the fractional-order memristor from the scope (XY Graph) in Figure 6(b). (a) The order $\alpha=0.98$ with two different frequencies; (b) the frequency $\omega=1 \mathrm{rad} / \mathrm{s}$ with three different fractional orders. 


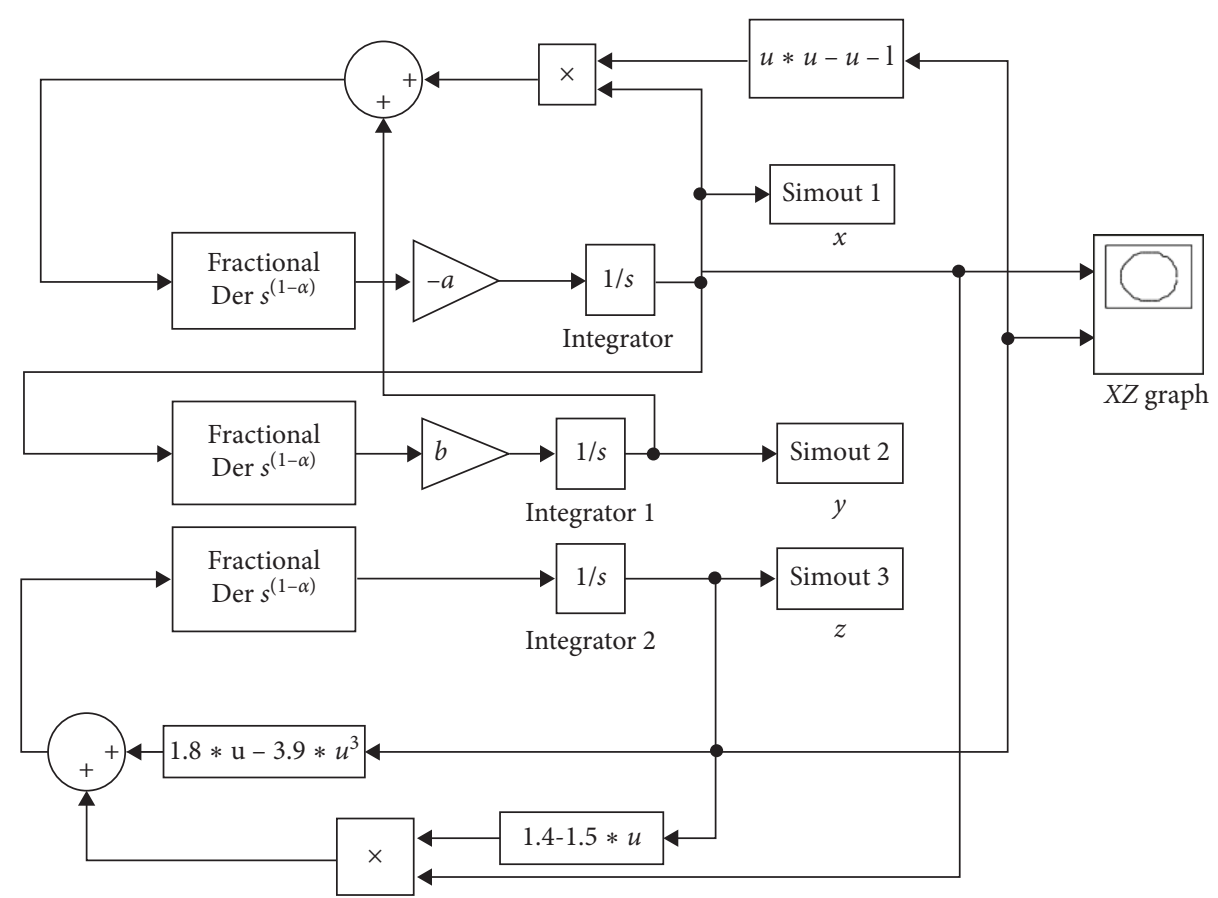

FIgure 8: The block diagram of system (5) in Simulink of MATLAB.

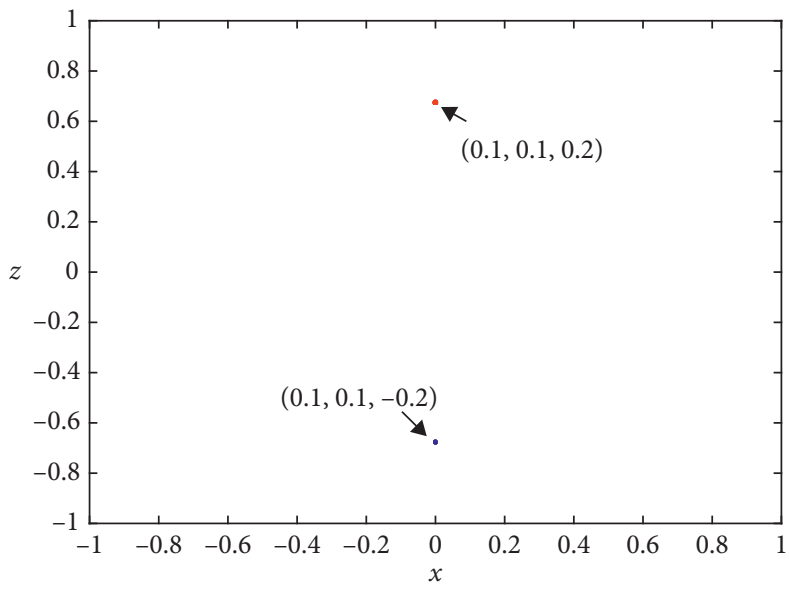

(a)

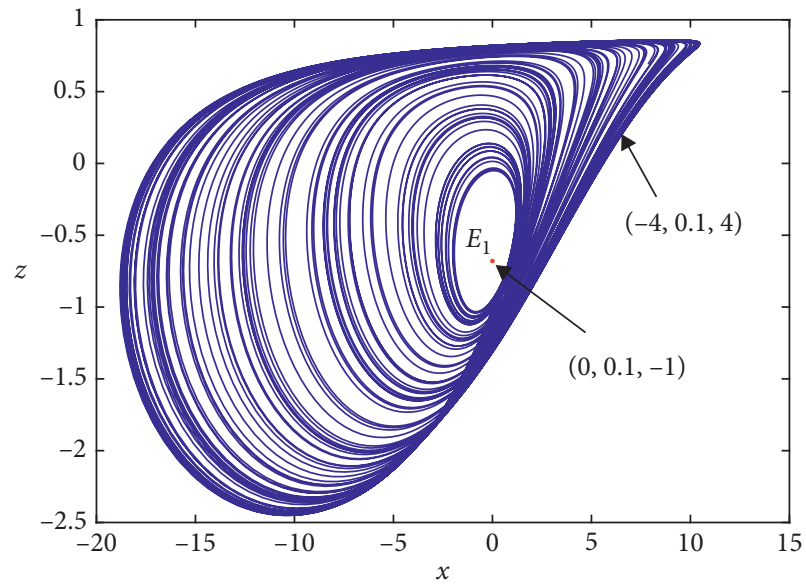

(b)

FIGURE 9: Bistability behaviors obtained in Simulink of MATLAB, the running time $t=100 \mathrm{~s}$. (a) $\alpha=0.75$, two sets of the initial values ( 0.1 , $0.1,0.2)$ and $(0.1,0.1,-0.2)$; (b) $\alpha=0.99$, two sets of the initial values $(-4,0.1,4)$ and $(0,0.1,-1)$.

To obtain the bistability behaviors of $\alpha=0.96$ and $\alpha=0.97$ in Simulink of MATLAB, the values of $(1-\alpha)$ in Figure 8 are set at $1-\alpha=0.04$ and $1-\alpha=0.03$, which are omitted here.

\section{Local Activeness and Stability}

A locally active kinetic equation can exhibit complex dynamics such as limit cycles or chaos. The passive (not locally active) kinetic equation must converge to a unique steady state [39]. Furthermore, the time $t$ can be set to be large when the steady states of the system are concerned.
As parameters $a=10 / 3$ and $b=10$, for the fractional-order memristor in system (5) with the large time $t$, one has the following.

Fix the initial values $(0.1,0.1,0.2)$. As $\alpha=0.75$, the memristor is active because the power $p=W_{0.75}\left(x_{m}\right) \cdot v^{2}$ keeps negative, which is shown in Figure 10(a); as $\alpha=0.82$, the memristor is passive because the power $p=W_{0.82}\left(x_{m}\right)$. $v^{2}$ keeps positive for time $t>t_{0}$ (such as $t_{0}=50$ ), which is shown in Figure 10(b). As mentioned before, system (5) with $\alpha=0.75$ or $\alpha=0.82$ converges to stable points. As $\alpha=0.9$ or $\alpha=0.99$, the memristor is locally active because the power $p=W_{\alpha}\left(x_{m}\right) \cdot v^{2}$ changes between the positive and the 


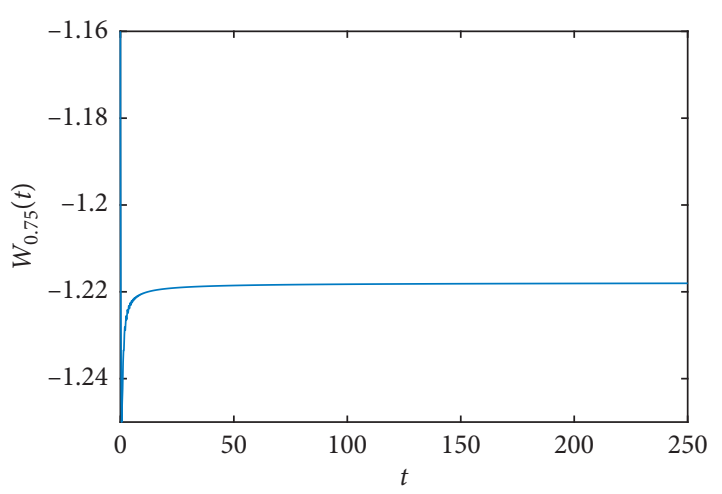

(a)

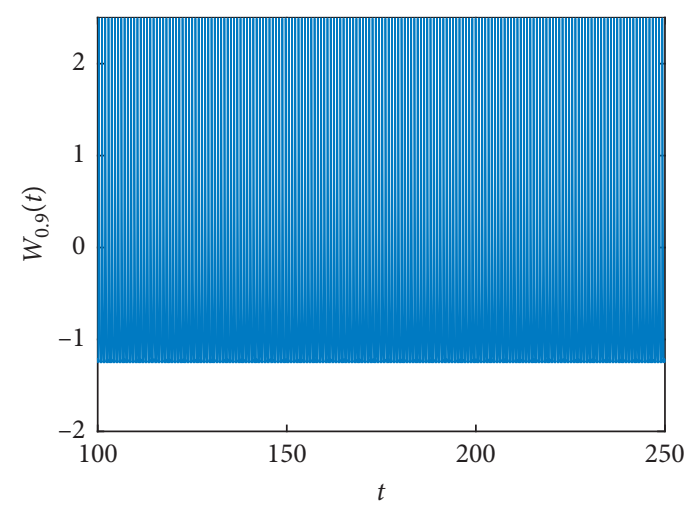

(c)

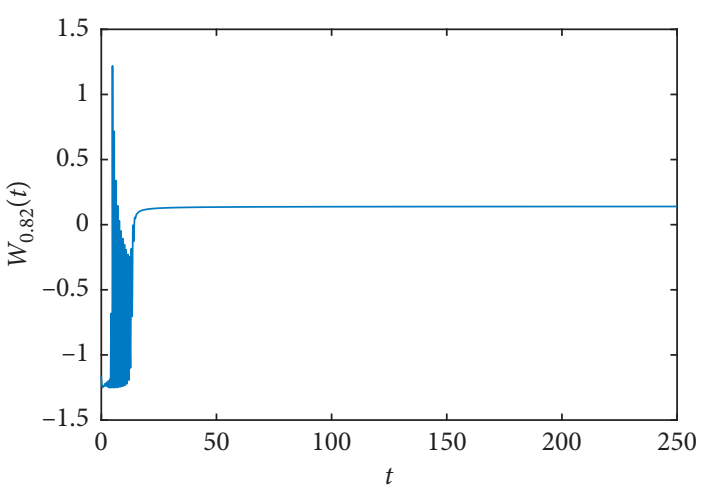

(b)

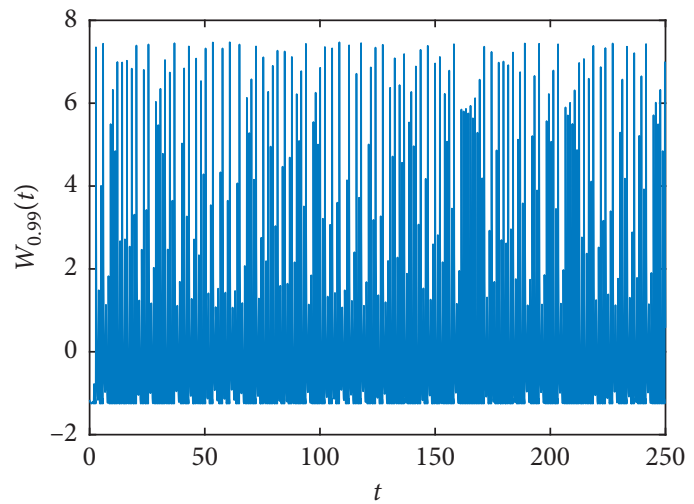

(d)

Figure 10: The memductance $W_{\alpha}(t)$ with initial values $(0.1,0.1,0.2)$. (a) Memductance $W_{0.75}(t)$; (b) memductance $W_{0.82}(t)$; (c) memductance $W_{0.9}(t)$; (d) memductance $W_{0.99}(t)$.

negative, as shown in Figures 10(c) and 10(d). When the locally active memristor is included in system (5), the limit circle is generated as $\alpha=0.9$ and chaos is generated as $\alpha=0.99$.

It is found that the complex dynamics of the limit cycle and chaos are not displayed in system (5) when the memristor is active $(\alpha=0.75)$ or the memristor is passive $(\alpha=0.82)$, while the complex dynamics of the limit cycle and the chaos are generated when the memristor is locally active $(\alpha=0.9$ and $\alpha=0.99$ ). As stated at the beginning of this section, complex dynamics of limit cycle and chaos in system (5) are related to the local activeness of the memristor.

\section{Conclusions}

In this paper, a chaotic system with a fractional-order locally active memristor is discussed. The fractional order in the memristive system makes the equilibrium vary from unstable to stable, leading to the occurrence of Hopf bifurcation. Moreover, the fractional-order memristive system enters into chaos via period-doubling bifurcation route and triggers more periodic windows than the corresponding integer-order system. Given the suitable parameters, say, $a$ and $b$, the fractional-order memristive system shows bistability behaviors. For different fractional order and different inductance, the fractional-order memristive system displays different bistability behaviors. The fractional order of the system and the local activeness of the memristor are the main reasons for the complicated dynamical behaviors. Besides, the fractional-order memristive system is implemented using the block diagram of Simulink of MATLAB and its hardware implementation and corresponding experiments will be our future works.

\section{Data Availability}

The data used to support the findings of this study are available from the corresponding author upon request.

\section{Conflicts of Interest}

The authors declare that they have no conflicts of interest.

\section{Acknowledgments}

This work was supported by the National Natural Science Foundations of China under Grant nos. 11602035, 11402125, 61601062, and 61801054 and the Natural Science Foundation of Jiangsu Province under Grant no. BK20191451.

\section{References}

[1] A. P. Kuznetsov, S. P. Kuznetsov, E. Mosekilde, and N. V. Stankevich, "Co-existing hidden attractors in a radiophysical system," Journal of Physics A: Mathematical and Theoretical, vol. 48, no. 12, Article ID 125101, 2015. 
[2] L. Chua, "Memristor-the missing circuit element," IEEE Transactions on Circuit Theory, vol. 18, no. 5, pp. 507-519, 1971.

[3] B. Bao, T. Jiang, Q. Xu, M. Chen, H. Wu, and Y. Hu, "Coexisting infinitely many attractors in active band-pass filter-based memristive circuit," Nonlinear Dynamics, vol. 86, no. 3, pp. 1711-1723, 2016.

[4] B. Muthuswamy and L. O. Chua, "Simplest chaotic circuit," International Journal of Bifurcation and Chaos, vol. 20, no. 5, pp. 1567-1580, 2010.

[5] H. Wu, B. Bao, Z. Liu, Q. Xu, and P. Jiang, "Chaotic and periodic bursting phenomena in a memristive Wien-bridge oscillator," Nonlinear Dynamics, vol. 83, no. 1-2, pp. 893-903, 2016.

[6] B.-C. Bao, M. Chen, H. Bao, and Q. Xu, "Extreme multistability in a memristive circuit," Electronics Letters, vol. 52, no. 12, pp. 1008-1010, 2016.

[7] Q. Li, H. Zeng, and J. Li, "Hyperchaos in a $4 \mathrm{D}$ memristive circuit with infinitely many stable equilibria," Nonlinear Dynamics, vol. 79, no. 4, pp. 2295-2308, 2015.

[8] N. H. Alombah, H. Fotsin, E. B. M. Ngouonkadi, and T. Nguazon, "Dynamics, analysis and implementation of a multi-scroll memristor-based chaotic circuit," International Journal of Bifurcation and Chaos, vol. 26, no. 8, Article ID 1650128, 2016.

[9] M. Kountchou, P. Louodop, S. Bowong, H. Fotsin, and J. Kurths, "Optimal synchronization of a memristive chaotic circuit," International Journal of Bifurcation and Chaos, vol. 26, no. 6, Article ID 1650093, 2016.

[10] Q. Xu, Y. Lin, B. Bao, and M. Chen, "Multiple attractors in a non-ideal active voltage-controlled memristor based Chua's circuit," Chaos, Solitons \& Fractals, vol. 83, pp. 186-200, 2016.

[11] B. C. Bao, P. Y. Wu, H. Bao, Q. Xu, and M. Chen, "Numerical and experimental confirmations of quasi-periodic behavior and chaotic bursting in third-order autonomous memristive oscillator," Chaos, Solitons \& Fractals, vol. 106, pp. 161-170, 2018.

[12] I. Petras, "Fractional-order memristor-based Chua's circuit," IEEE Transactions on Circuits and Systems II: Express Briefs, vol. 57, no. 12, pp. 975-979, 2010.

[13] J. Kengne, G. D. Leutcho, and A. N. Kengnou Telem, "Reversals of period doubling, coexisting multiple attractors, and offset boosting in a novel memristive diode bridge-based hyperjerk circuit," Analog Integrated Circuits and Signal Processing, pp. 1-21, 2018.

[14] J. Ma, Z. Chen, Z. Wang, and Q. Zhang, "A four-wing hyperchaotic attractor generated from a 4-D memristive system with a line equilibrium," Nonlinear Dynamics, vol. 81, no. 3, pp. 1275-1288, 2015.

[15] L. Zhou, C. Wang, and L. Zhou, "Generating hyperchaotic multi-wing attractor in a 4D memristive circuit," Nonlinear Dynamics, vol. 85, no. 4, pp. 2653-2663, 2016.

[16] J. Kengne, Z. Njitacke Tabekoueng, V. Nguomkam Negou, and A. Negou, "Periodicity, chaos, and multiple attractors in a memristor-based Shinriki's circuit," Chaos: An Interdisciplinary Journal of Nonlinear Science, vol. 25, no. 10, Article ID 103126, 2015.

[17] Z. T. Njitacke, J. Kengne, H. B. Fotsin, A. N. Negou, and D. Tchiotsop, "Coexistence of multiple attractors and crisis route to chaos in a novel memristive diode bridge-based Jerk circuit," Chaos Solitons \& Fractals, vol. 91, pp. 180-197, 2016.

[18] P. Saha, D. C. Saha, A. Ray, and A. R. Chowdhury, "Memristive non-linear system and hidden attractor," The European Physical Journal Special Topics, vol. 224, no. 8, pp. 1563-1574, 2015.
[19] B. C. Bao, A. H. Hu, H. Bao, Q. Xu, M. Chen, and H. G. Wu, "Three-dimensional memristive hindmarsh-rose neuron model with hidden coexisting asymmetric behaviors," Complexity, vol. 2018, Article ID 3872573, 11 pages, 2018.

[20] A. I. Ahamed and M. Lakshmanan, "Non-smooth bifurcations, transient hyper-chaos and hyperchaotic beats in a memristive Murali-Lakshmanan-Chua circuit," International Journal of Bifurcation and Chaos, vol. 23, no. 6, Article ID 1350098, 2013.

[21] R. Barboza and L. O. Chua, "The four-element Chua's circuit," International Journal of Bifurcation and Chaos, vol. 18, no. 4, pp. 943-955, 2008.

[22] D. B. Strukov, G. S. Snider, D. R. Stewart, and R. S. Williams, "The missing memristor found," Nature, vol. 453, no. 7191, pp. 80-83, 2008.

[23] I. Podlubny, Fractional Differential Equations, Academic Press, Cambridge, MA, USA, 1999.

[24] Y. J. Yu and Z. H. Wang, "A fractional-order memristor model and the fingerprint of the simple series circuits including a fractional-order memristor," Acta. Phys. Sinica, vol. 64, no. 23, Article ID 238401, 2015.

[25] M. E. Fouda and A. G. Radwanon, "The fractional-order memristor model," Journal of Fractional Calculus and Applications, vol. 4, no. 1, pp. 1-7, 2013.

[26] D. Cafagna and G. Grassi, "On the simplest fractional-order memristor-based chaotic system," Nonlinear Dynamics, vol. 70, no. 2, pp. 1185-1197, 2012.

[27] M.-F. Danca, W. K. S. Tang, and G. Chen, "Suppressing chaos in a simplest autonomous memristor-based circuit of fractional order by periodic impulses," Chaos, Solitons \& Fractals, vol. 84, pp. 31-40, 2016.

[28] R. Dogaru and L. O. Chua, "Edge of chaos and local activity domain of FitzHugh-Nagumo equation," International Journal of Bifurcation and Chaos, vol. 8, no. 2, pp. 211-257, 1998.

[29] R. Dogaru and L. O. Chua, "Edge of chaos and local activity domain of the Brusselator CNN," International Journal of Bifurcation and Chaos, vol. 8, no. 6, pp. 1107-1130, 1998.

[30] P. Jin, G. Wang, H. H.-C. Iu, and T. Fernando, "A locally active memristor and its application in a chaotic circuit," IEEE Transactions on Circuits and Systems II: Express Briefs, vol. 65, no. 2, pp. 246-250, 2018.

[31] D. Biolek, V. Biolková, and Z. Biolek, "Interpreting area of pinched memristor hysteresis loop," Electronics Letters, vol. 50, no. 2, pp. 74-75, 2014.

[32] S. Westerlund and L. Ekstam, "Capacitor theory," IEEE Transactions on Dielectrics and Electrical Insulation, vol. 1, no. 5, pp. 826-839, 1994.

[33] E. Ahmed, A. M. A. El-Sayed, and H. A. A. El-Saka, "Equilibrium points, stability and numerical solutions of fractionalorder predator-prey and rabies models," Journal of Mathematical Analysis and Applications, vol. 325, no. 1, pp. 542-553, 2007.

[34] H. A. El-Saka, E. Ahmed, M. I. Shehata, and A. M. A. El-Sayed, "On stability, persistence, and Hopf bifurcation in fractional order dynamical systems," Nonlinear Dynamics, vol. 56, no. 1-2, pp. 121-126, 2009.

[35] L. Chua, M. Komuro, and T. Matsumoto, "The double scroll family," IEEE Transactions on Circuits and Systems, vol. 33, no. 11, pp. 1072-1118, 1986.

[36] M.-F. Danca and N. Kuznetsov, "Matlab code for Lyapunov exponents of fractional-order systems," International Journal of Bifurcation and Chaos, vol. 28, no. 5, Article ID 1850067, 2018. 
[37] C. A. Monje, Y. Q. Chen, B. M. Vinagre, D. Y. Xue, and V. Feliu, Fractional-Order Systems and Controls: Fundamentals and Applications, Springer-Verlag, London, UK, 2010.

[38] C. Li and W. Deng, "Remarks on fractional derivatives," Applied Mathematics and Computation, vol. 187, no. 2, pp. 777-784, 2007.

[39] L. O. Chua, "Local activity is the origin of complexity," International Journal of Bifurcation and Chaos, vol. 15, no. 11, pp. 3435-3456, 2005. 


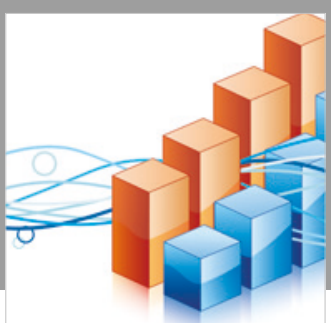

Advances in

Operations Research

\section{-n-m}
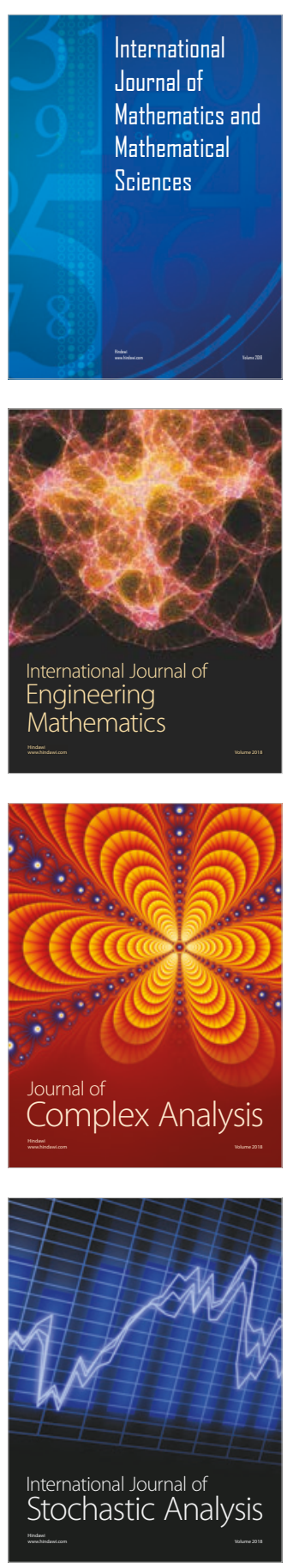
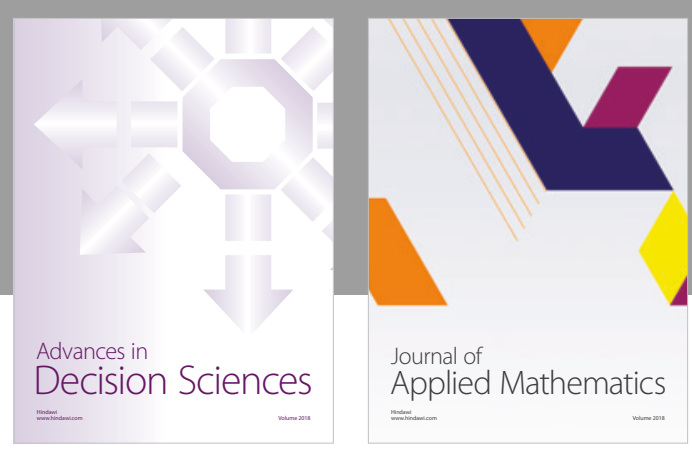

Journal of

Applied Mathematics
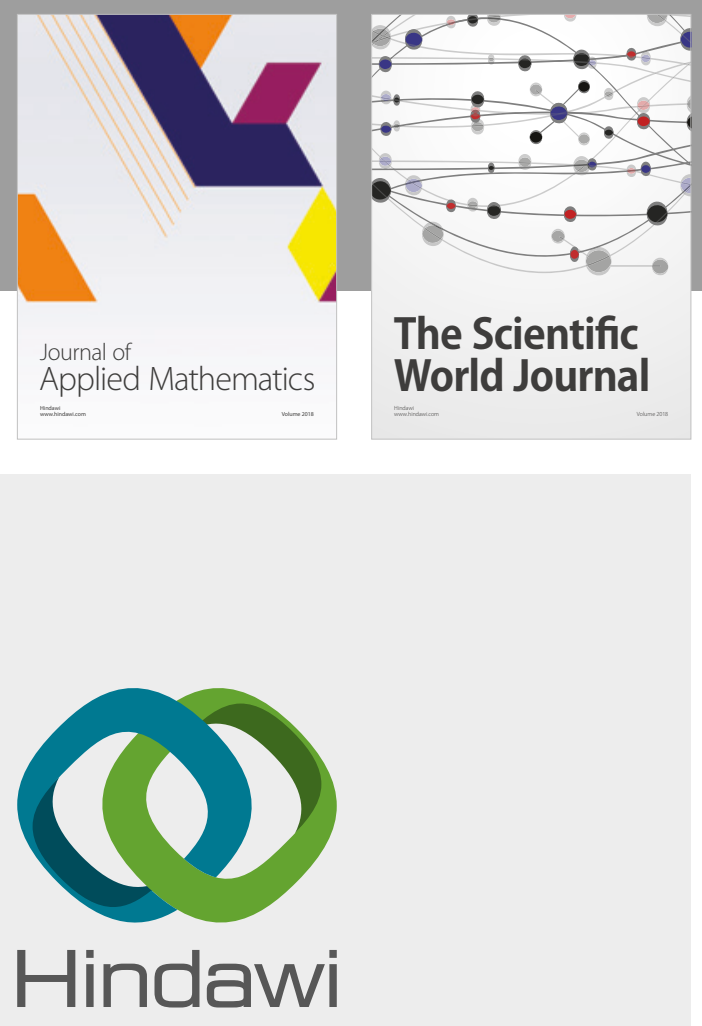

Submit your manuscripts at

www.hindawi.com

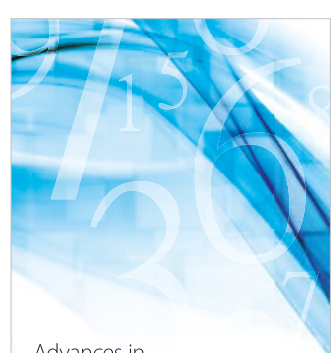

Advances in
Numerical Analysis
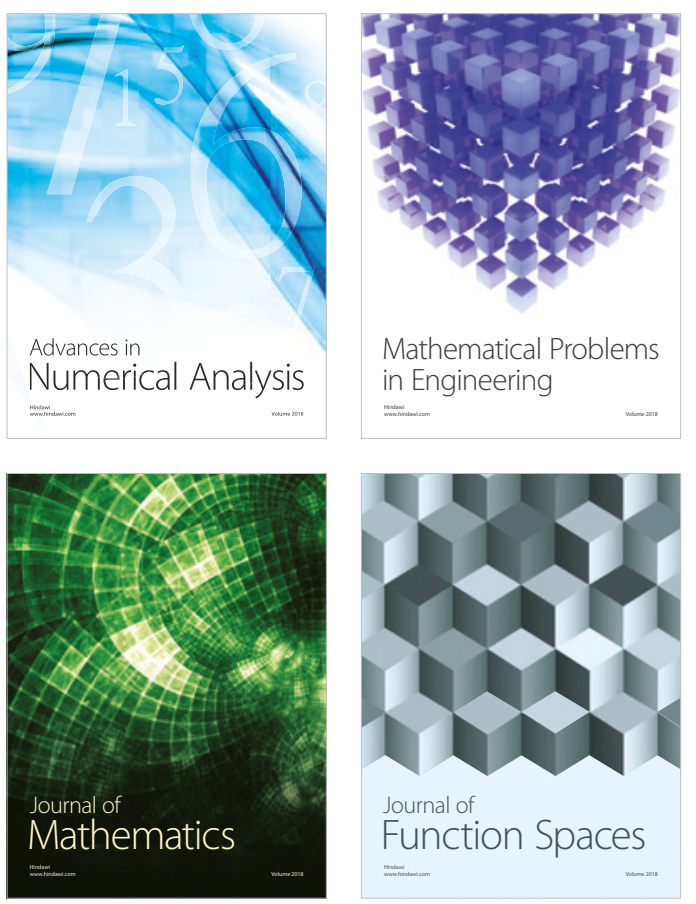

Mathematical Problems in Engineering

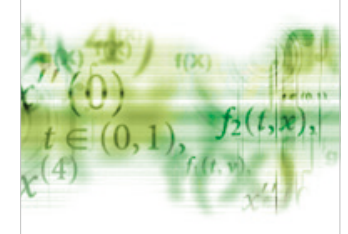

International Journal of

Differential Equations

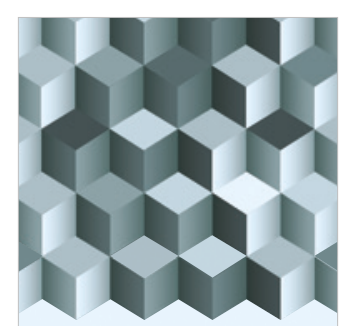

Journal of

Function Spaces
The Scientific

World Journal

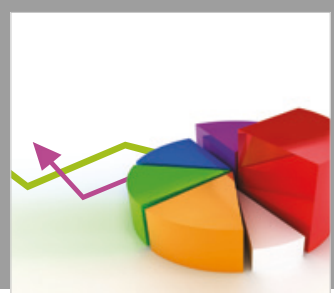

Journal of

Probability and Statistics
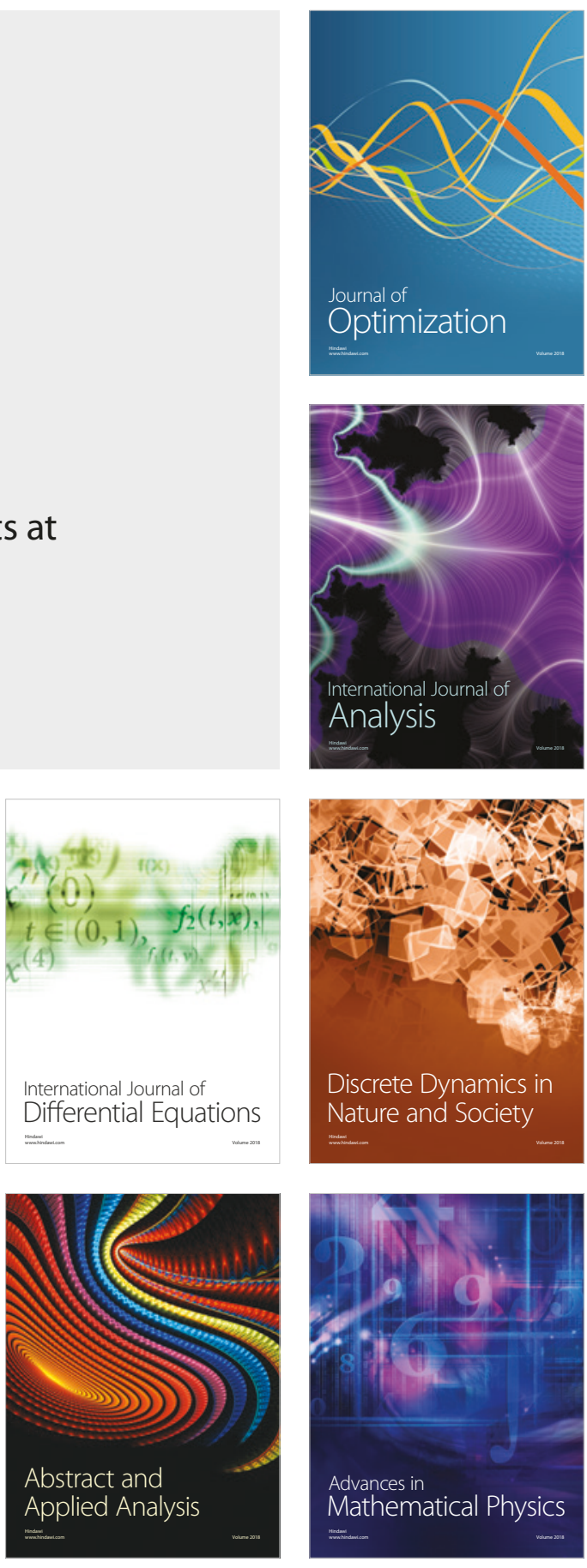